\title{
TITLE:
}

\section{Optimal Initial Capital Induced by the Optimized Certainty Equivalent}

$\operatorname{AUTHOR}(\mathrm{S})$ :

Arai, Takuji; Asano, Takao; Nishide, Katsumasa

\section{CITATION:}

Arai, Takuji ...[et al]. Optimal Initial Capital Induced by the Optimized Certainty Equivalent. KIER Discussion Paper 2017, 981: 1-20

ISSUE DATE:

2017-11-27

URL:

http://hdl.handle.net/2433/228366

RIGHT: 


\title{
KIER DISCUSSION PAPER SERIES
}

\author{
KYOTO INSTITUTE \\ OF \\ ECONOMIC RESEARCH
}

\author{
Discussion Paper No.981 \\ "Optimal Initial Capital Induced by the Optimized \\ Certainty Equivalent"
}

Takuji Arai, Takao Asano, and Katsumasa Nishide

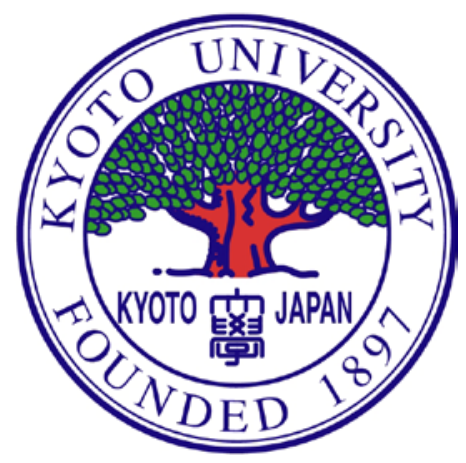

KYOTO UNIVERSITY

KYOTO, JAPAN 


\title{
Optimal Initial Capital Induced by the Optimized Certainty Equivalent*
}

\author{
Takuji Arai $^{\dagger}$, Takao Asano ${ }^{\ddagger}$, and Katsumasa Nishide ${ }^{\S}$
}

November 27, 2017

\begin{abstract}
This paper proposes the notion of optimal initial capital (OIC) induced by the optimized certainty equivalent (OCE) discussed in Ben-Tal and Teboulle (1986) and Ben-Tal and Teboulle (2007), and investigates the properties of the OIC with various types of utility functions. By providing its several properties with different utility functions or other assumptions, we successfully present the OIC as a monetary utility function (negative value of risk measure) for future payoffs with the decisionmaker's concrete criteria in the background.
\end{abstract}

Keywords: optimal initial capital, optimized certainty equivalence, monetary utility function, prudence premium.

JEL classification: D81, G32, G11, D46,

${ }^{*}$ We are grateful to Shigeo Kusuoka for his helpful comments. This research is partially supported by the MEXT Grant in Aid for Scientific Research (A) \#25245046, (A) \#16H02026, (B) \#15H02965, (B) \#16H03619, (C) \#17K03797, (C) \#15K04936, (C) \#16K03558, (C) \#17K03806, and the Joint Research Program of KIER, Kyoto University.

$\dagger$ Department of Economics, Keio University, 2-15-45 Mita, Minato-ku, Tokyo, 108-8345, Japan. Phone: (81)-3-5427-1411. E-mail: arai@econ.keio.ac.jp,

$¥$ Department of Economics, Okayama University, 3-1-1, Tsushimanaka, Kita-ku, Okayama 700-8530, Japan. Phone: (81)-86-251-7553. E-mail: asano@e.okayama-u.ac.jp.

$\S$ Graduate School of Economics, Hitotsubashi University. 2-1 Naka, Kunitachi, Tokyo 186-8601, Japan. E-mail: k.nishide@r.hit-u.ac.jp. 


\section{Introduction}

In economics, management, or problems in other areas entailing optimizations, uncertainty must be taken into consideration in the process of decision-making. The decisionmaker should take a one-dimensional action such as the choice to do or not, or choose one action among multiple alternatives. However, uncertainty has multi-dimensional aspects such as expectation, variance, kurtosis, and so on. This fact indicates the need to transform the multi-dimensionality of uncertainty into a one-dimensional value. The risk measure studied in the finance literature is one typical example of research objects with this motivation.

Ben-Tal and Teboulle (1986) propose the optimized certainty equivalent (OCE), which Ben-Tal and Teboulle (2007) later develop. It is regarded as an important decision theoretic criterion. Formally, the OCE for the random payoff $X$ with utility function $u$ is defined by

$$
S_{u}(X)=\sup _{t \in \mathbb{R}}\{t+\mathbb{E}[u(X-t)]\}
$$

It is interpreted as the maximized utility level when the decision maker with utility function $u$ allocates the amount of money $t$ in the current period to have an uncertain payoff $X-t$ in the future period. In other words, the OCE is the decision maker's optimized utility value from uncertain monetary incomes by the intertemporal allocation.

Many studies examine the OCE, which is of especially great significance in finance because it has the property of translation-invariance. In other words, the OCE is a monetary utility function, implying a close relationship to the risk measure studied in Artzner et al. (1999). Drapeau and Kupper (2013) investigate the OCE and show that the OCE formulation can represent the mean-variance risk measure, the conditional value-at-risk, and the entropic risk measure. Recently, Eeckhoudt et al. (2016) find a link between the OCE and the prudence premium in the sense of Kimball (1990). ${ }^{1}$ Other papers studying the OCE include those by Cheridito and Li (2009), Schied et al. (2009), Chen et al. (2010), and Goh and Sim (2010).

In this paper, we propose a new notion, the optimal initial capital (OIC), induced by the OCE. Roughly speaking, the OIC is the optimal amount of the initial allocation $t$ in (1.1). The rationale behind the OIC is similar to that of the OCE, but the concept is slightly different: the OCE is the evaluation for both the current and future periods when the decision maker faces a random payoff $X$ in the future period, while the OIC is the evaluation for only the current period. Put differently, the OIC is a decision maker's control variable for the allocation instead of the maximized utility level as in the OCE. One reason we consider the OIC rather than the OCE is that the OIC always quantifies the monetary value associated with the random payoff $X$, while the OCE does not if the utility function $u$ represents ordinal preferences. Another reason is that we can cover a broader class of utility functions $u$ with the OIC than with the OCE, as we discuss later.

\footnotetext{
${ }^{1}$ The model constructed in Ben-Tal and Teboulle (1986) is very similar to the models in Pratt (1964) and Kimball (1990).
} 
The OIC is especially important in the insurance industry. Suppose the situation where the insurance company faces the problem of how much amount of money to collect from the policy purchaser as a premium. Then, as we will see later, the optimal amount should be described by the OIC. Therefore, the properties of the OIC are worth studying in depth from the viewpoint of insurance economics. ${ }^{2}$

In the current study, we thoroughly examine the OIC with the same research motivation as extant studies of the OCE. More specifically, we present the properties of the $\mathrm{OIC}$, depending on the functional form of the utility functions for the future random payoff within a unified framework. We consider several utility functions: piecewise-linear, quadratic, exponential, and of power-type. By investigating the OIC with various utility functions, we show that the OIC is indeed an important monetary utility function (negative value of risk measure) for uncertain future payoffs and portfolios.

The major findings of the study are as follows. First, we show that the OIC is a lawinvariant monetary utility function when it fulfills the condition that $u^{\prime}$, the first derivative of the utility function in (1.1), is convex. This finding implies that the OIC is appropriate for a functional to express insurance premiums, or for a monetary utility function for financial products and portfolios in a wide class of utility functions. ${ }^{3}$ The finding also demonstrates that the OIC can define the risk measure for broader preferences for the financial risk. Moreover, we present a counterexample for the OIC to be a law-invariant monetary utility function.

Second, the OIC represents a notion of prudence, which is often studied in the microeconomics literature. More precisely, the OIC is essentially the same as the modified prudence premium defined in Eeckhoudt et al. (2016). Prudence describes how a decision maker saves money for uncertain future incomes as a precautionary reason, and is characterized by the third derivative of a utility function. Our observation indicates that the risk measure is closely related to precaution in the microeconomics. Note that we can conjecture the importance of the third derivative of $u$ in (1.1) by the convexity of $u^{\prime}$ as we mentioned in the previous paragraph.

Third, we make some analytical comparisons between the OIC and OCE with various utility functions. One important finding is that the coherency of the OCE is a necessary and sufficient condition for the OIC to be coherent under some conditions. Moreover, we show that the exponential utility is the only case where the OIC and OCE coincide. We also show that if the OIC is coherent, the OCE is precisely equal to the expectation of the payoff $X$ if $u^{\prime}$ is convex.

In summary, by providing several properties of the OIC with different utility functions or other assumptions, we successfully present the OIC as a monetary utility function for future payoffs with the decision-maker's concrete criteria in the background. This is the main contribution of our study to the literature.

The rest of the paper is organized as follows. We present some preliminary results for

\footnotetext{
${ }^{2}$ Recent studies of risk measures in insurance economics include Boonen (2015), Kong et al. (2017), Liebrich and Svindland (2017), and Rieger (2017). Based on risk measures, Balbás et al. (2009), Balbás et al. (2015), Tan et al. (2011), and Chi and Tan (2013) investigate optimal reinsurance problems.

${ }^{3}$ Refer to Chapter 4 of Föllmer and Schied (2016) as the discussions on the topic.
} 
our analysis in Section 2. Following Ben-Tal and Teboulle (2007), we focus on discussing the case where the utility function is piecewise linear in Subsection 2.1. We examine the properties of the OIC in the case of quadratic and exponential utility functions in Section 3. In this section, we also derive some conditions for the OIC to be coherent. In Section 4, we examine the OIC in the case of power and logarithmic utility functions, and show that the OIC becomes a law-invariant monetary utility function. Concluding remarks of this study are presented in Section 5.

\section{Preliminaries}

Let $\Omega$ be a set of possible scenarios, and $(\Omega, \mathscr{F}, \mathbb{P})$ a probability space, where $\mathscr{F}$ is a $\sigma$ algebra on $\Omega$ and $\mathbb{P}$ is a probability measure on $\mathscr{F}$. Suppose that $(\Omega, \mathscr{F}, \mathbb{P})$ is atomless, that is, for every $F \in \mathscr{F}$ with $\mathbb{P}(F)>0$, there exists a set $E \in \mathscr{F}$ such that $E \subset F$ and $0<\mathbb{P}(E)<\mathbb{P}(F)$. Let $L^{\infty}(\Omega, \mathscr{F}, \mathbb{P})$ be the space of all equivalence classes with respect to the equivalence relation defined by $f \sim g \Leftrightarrow f=g \mathbb{P}$-a.s. for $f, g \in \mathscr{L}^{\infty}(\Omega, \mathscr{F}, \mathbb{P})$, where $\mathscr{L}^{\infty}(\Omega, \mathscr{F}, \mathbb{P})$ denotes the set of all $\mathscr{F}$-measurable bounded functions on $(\Omega, \mathscr{F}, \mathbb{P})$. For ease of notation, $L^{\infty}(\Omega, \mathscr{F}, \mathbb{P})$ is written by $L^{\infty}$.

Let $u: \mathbb{R} \rightarrow[-\infty,+\infty)$ be the utility function for the future payoff, and assume the following conditions:

\section{Assumption 2.1. $\quad$ (i) $u$ is closed concave and non-decreasing,}

(ii) $u(0)=0$ and $1 \in \partial u(0)$, where $\partial u(\cdot)$ denotes the subdifferential map of $u$,

(iii) the effective domain is given by $\operatorname{dom} u=\{t \in \mathbb{R} \mid u(t)>-\infty\} \neq \emptyset$.

Note that for any utility function $u$ in this paper, we have $u(t) \leq t$ for any $t \in \mathbb{R}$, and $u(t) \geq 0$ for any $t>0$.

For the purpose of quantifying the risk associated with random payoffs such as stock prices or interest rates, several risk measures have been proposed. In the literature, Artzner et al. (1999) is the seminal paper proposing the coherent risk measures. After their paper, as important extensions and generalizations, Acerbi and Tasche (2002) and Föllmer and Schied (2002) propose convex risk measures, which cover the coherent risk measures as a special case. ${ }^{4}$ Finally, the monetary risk measures are risk measures that do not necessarily have the property of convexity.

Formally, a function $\rho: L^{\infty} \rightarrow \mathbb{R}$ is called a monetary risk measure if the following conditions hold for any $X, Y \in L^{\infty}$ :

1. (Monotonicity) If $X \leq Y$ a.s., then $\rho(X) \geq \rho(Y)$.

2. (Translation-Invariance) If $m \in \mathbb{R}$, then $\rho(X+m)=\rho(X)-m$.

\footnotetext{
${ }^{4}$ See also Frittelli and Rosazza Gianin (2002), Föllmer and Schied (2016), and the references therein.
} 
Monotonicity states that if the future payoff increases almost surely, then the measured risk should decrease. Translation-Invariance means that when the amount $m$ of a risk-free asset is added to the position, the measured risk should be reduced exactly by the amount $m$. A monetary risk measure $\rho: L^{\infty} \rightarrow \mathbb{R}$ is called a convex risk measure if $\rho(\lambda X+(1-$ $\lambda) Y) \leq \lambda \rho(X)+(1-\lambda) \rho(Y)$ for any $\lambda \in[0,1]$ and any $X, Y \in L^{\infty}$. Convexity states that the convex risk measure successfully describes the risk diversification effect. A convex risk measure $\rho: L^{\infty} \rightarrow \mathbb{R}$ is called a coherent risk measure if the positive homogeneity holds, i.e., $\rho(\lambda X)=\lambda \rho(X)$ for any $\lambda \geq 0$ and any $X \in L^{\infty}$. Coherency states that the risk of a financial position increases linearly as the size of the position increases. Finally, a functional $\eta$ is a concave monetary utility function if it has a representation

$$
\eta(X)=-\rho(X)
$$

with a convex risk measure $\rho$. Hereafter we simply call this $\eta$ a monetary utility function.

Next, we consider the relationship between risk measures and preference orders, $\succeq$. Let $X, Y$ be random variables. A risk measure $\rho$ quantifies the risk of random variables, namely, $X \succeq Y$ if and only if $\rho(X) \leq \rho(Y)$. In the expected utility theory, $X \succeq Y$ if and only if $\mathbb{E}[u(X)] \geq \mathbb{E}[u(Y)]$, where $u$ denotes the decision maker's utility function. Moreover, if $u$ is strictly increasing, then $X \succeq Y$ if and only if $C_{u}(X) \geq C_{u}(Y)$, where $C_{u}(Z):=u^{-1} \mathbb{E}[u(Z)]$ is the certainty equivalent for a random variable $Z$.

For utility function $u$, the OCE, originally proposed by Ben-Tal and Teboulle (1986), is defined as the functional $S_{u}: L^{\infty} \rightarrow \mathbb{R}$,

$$
S_{u}(X):=\sup _{t \in \mathbb{R}}\{t+\mathbb{E}[u(X-t)]\} .
$$

As Ben-Tal and Teboulle (2007) show, the OCE has the following properties:

1. For any utility function $u$, the OCE $S_{u}$ is a law-invariant monetary utility function, where a monetary utility function $U$ is called law-invariant if $U(X)=U(Y)$ whenever $X$ and $Y$ have the same distribution under $\mathbb{P}$.

2. The OCE $S_{u}$ is consistent, that is, $S_{u}(c)=c$ for any $c \in \mathbb{R}$.

3. (Theorem 3.1 of Ben-Tal and Teboulle, 2007) When $u$ is a utility function such that $u(t)<t$ for any $t \in \mathbb{R} \backslash\{0\}$, the OCE $S_{u}$ is coherent, that is,

$$
S_{u}(\lambda X)=\lambda S_{u}(X)
$$

for any $\lambda \geq 0$ and any $X \in L^{\infty}$, if and only if $u$ is a piecewise linear function defined as follows:

$$
u(t)= \begin{cases}\gamma_{1} t, & \text { if } t \geq 0, \\ \gamma_{2} t, & \text { if } t<0,\end{cases}
$$

where $0 \leq \gamma_{1}<1<\gamma_{2}<\infty$. 
In the following, we denote the piecewise linear function in (2.3) by $u_{\gamma_{1}, \gamma_{2}}$.

Some comments are in order. First, regarding the relationship between the OCE $S_{u}$ and the certainty equivalent $C_{u}$, it is worth mentioning that the OCE induces the same order that the certainty equivalent does. ${ }^{5}$ Second, as Ben-Tal and Teboulle (1986) show, whether the risk measure $\Gamma(X):=-C_{u}(X)$ is concave or coherent depends on the shape of the utility functions. More specifically, let $u$ be a $C^{3}$-class function with $u^{\prime}>0$ and $u^{\prime \prime}<0$. Then, the certainty equivalent $C_{u}$ is concave if and only if $1 / r$ is concave, where $r: \mathbb{R} \rightarrow \mathbb{R}$ defined by $r(t)=-u^{\prime \prime}(t) / u^{\prime}(t)$ is the Arrow-Pratt measure of absolute risk aversion. As is well known in the literature, a class of utility functions satisfying the concavity of $1 / r$ is the class of hyperbolic absolute risk aversion (HARA) defined by $r(t)=1 /(a t+b)$ for $t>-b / a$. Here, a utility function in the class of HARA is represented by

$$
u(t)= \begin{cases}b\left(1-e^{-t / b}\right) & \text { if } \quad a=0, b \neq 0 \\ \log (b+t) & \text { if } a=1 \\ (a t+b)^{(a-1) / a} & \text { if } \quad a \neq 0, a \neq 1\end{cases}
$$

Ben-Tal and Teboulle (2007) also show that $\Gamma(X)=-C_{u}(X)$ is a convex risk measure only for the class of exponential utilities $u_{b}(t):=b\left(1-e^{-t / b}\right)$ for $0<b<\infty$, and that $\Gamma(X)=-C_{u}(X)$ is a coherent risk measure only for the linear utility $u(t)=t$.

We are now ready to propose a new notion, which is the main research object in this study. For a given $X \in L^{\infty}$, even if there exists a $t^{*} \in \mathbb{R}$ such that

$$
S_{u}(X)=t^{*}+\mathbb{E}\left[u\left(X-t^{*}\right)\right],
$$

this $t^{*}$ is not necessarily unique. Therefore, we take the supremum and write $\eta_{u}^{*}(X)$ for a random payoff $X$. In other words, we define $\eta_{u}^{*}(X)$ by

$$
\eta_{u}^{*}(X):=\sup \left\{t \in \mathbb{R} \mid S_{u}(X)=t+\mathbb{E}[u(X-t)]\right\},
$$

where $\eta_{u}^{*}(X)=-\infty$ if the set $\left\{t \in \mathbb{R} \mid S_{u}(X)=t+\mathbb{E}[u(X-t)]\right\}=\emptyset$. We call this $\eta_{u}^{*}$ the optimal initial capital (OIC) corresponding to $u$ (or $S_{u}$ ). Roughly speaking, we can rewrite (2.4) as $S_{u}\left(X-\eta_{u}^{*}(X)\right)=\mathbb{E}\left[u\left(X-\eta_{u}^{*}(X)\right)\right]$ since $S_{u}$ is a monetary utility function. As we discuss in Section 3, $\eta_{u}^{*}(X)$ is a real number that attains the supremum on the right-hand-side of (2.2), and satisfies $\mathbb{E}\left[u^{\prime}\left(X-\eta_{u}^{*}(X)\right)\right]=1$ if $u$ is a $C^{1}$-class function.

The motivation to study the OIC is almost the same as the OCE. That is, the OIC is derived from a decision-making problem in the two stage setting, in which the decision maker receives the amount $t$ at the current period, and $X-t$ at the future period. The preference of the random payoff $X-t$ is described by the expected utility given in (2.2). The difference of the OIC from the OCE is that the OIC is the monetary value at the current period, while the OCE is the evaluation for both the current and future periods. Mathematically, the OIC is the optimal control variable at the initial stage, and the OCE is the optimized utility value for the problem (2.2).

\footnotetext{
${ }^{5}$ For any utility function $u, S_{u}(X) \geq S_{u}(Y)$ if and only if $C_{u}(X) \geq C_{u}(Y)$. See Proposition 2.4 in Ben-Tal and Teboulle (2007).
} 
Intuitively, the OCE is the optimized value for both the initial and future periods, while the OIC is the optimal amount of initial reserve or capital for the future random payoffs. For example, in insurance economics, the OIC indicates the optimal insurance premium for each random claim as discussed in the introductory section. Therefore, it is worth studying the OIC in depth, especially in insurance economics.

To use the OIC as an evaluation method for the random payoff of a financial product, we need to check whether or not the OIC is appropriate for our purpose. Especially, it is of great importance to examine the conditions for the OIC to be a monetary utility function in the literature on risk measures. In the following, we conduct a thorough analysis on the OIC with a wide variety of utility functions within a unified framework. We also make some analytical comparisons of the OIC and OCE to clarify the difference between the two functionals.

\subsection{OIC for Piecewise Linear Utility Functions}

In this subsection, following the analysis by Ben-Tal and Teboulle (2007), we discuss the piecewise linear OCE and the piecewise linear OIC for the utility function $u_{\gamma_{1}, \gamma_{2}}$ in (2.3). We denote the OCE and OIC for $u_{\gamma_{1}, \gamma_{2}}$ by $S_{\gamma_{1}, \gamma_{2}}$ and $\eta_{\gamma_{1}, \gamma_{2}}$, respectively. As we see later, the piecewise linear OCE turns out to be coherent risk measure, but the OIC does not.

For $\alpha \in(0,1]$, we define a functional $\mathrm{CVaR}_{\alpha}$ by

$$
\operatorname{CVaR}_{\alpha}(X):=\frac{1}{\alpha} \int_{-\infty}^{F_{X}^{-1}(\alpha)} t d F_{X}(t)
$$

where $F_{X}$ is the distribution function of a random variable $X$, and $F_{X}^{-1}$ is the rightcontinuous inverse function of $F_{X}$, that is, $F_{X}^{-1}(s):=\inf \left\{t \mid F_{X}(t)>s\right\}$. We can easily verify that $\mathrm{CVaR}_{\alpha}$ is a law-invariant coherent monetary utility function. Note that this $\mathrm{CVaR}_{\alpha}$ corresponds to the usual $\mathrm{CVaR}_{\alpha}$ as a coherent risk measure multiplied by -1 . In addition, we have $\mathrm{CVaR}_{1}(X)=\mathbb{E}[X]$, and $\lim _{\alpha \rightarrow 0} \mathrm{CVaR}_{\alpha}(X)=\operatorname{essinf} X$.

Denoting $\Gamma:=\left\{\left(\gamma_{1}, \gamma_{2}\right) \mid 0 \leq \gamma_{1}<1<\gamma_{2}<\infty\right\}$ and $\delta:=\left(1-\gamma_{1}\right) /\left(\gamma_{2}-\gamma_{1}\right)$ for $\left(\gamma_{1}, \gamma_{2}\right) \in \Gamma$, we obtain

$$
\begin{aligned}
S_{\gamma_{1}, \gamma_{2}}(X) & =\gamma_{2} \int_{-\infty}^{F_{X}^{-1}(\delta)} t d F_{X}(t)+\gamma_{1} \int_{F_{X}^{-1}(\delta)}^{\infty} t d F_{X}(t) \\
& =\left(1-\gamma_{1}\right) \operatorname{CVaR}_{\delta}(X)+\gamma_{1} \mathbb{E}[X] .
\end{aligned}
$$

That is, the law-invariant coherent monetary utility function $S_{\gamma_{1}, \gamma_{2}}$ is described by a convex combination of $\mathbb{E}[X]$ and $\mathrm{CVaR}_{\alpha}(X)$. Furthermore, by taking the limits of $\gamma_{1}$ and $\gamma_{2}$, letting $0 / 0=1$, and expanding the set $\Gamma$, we can define

$$
\begin{aligned}
S_{\gamma_{1}, \infty}(X) & :=\left(1-\gamma_{1}\right) \operatorname{essinf} X+\gamma_{1} \mathbb{E}[X], \text { for } \gamma_{1} \in[0,1), \\
S_{1,1}(X) & :=\mathbb{E}[X] .
\end{aligned}
$$

In particular, $S_{0, \infty}(X)=\operatorname{essinf} X$. Like $S_{\gamma_{1}, \gamma_{2}}$, the two functionals above are also lawinvariant coherent monetary utility functions, and can be considered to be piecewise linear 
OCEs in a broad sense. Note that the concave utility function $u_{\gamma_{1}, \infty}$ corresponding to $S_{\gamma_{1}, \infty}$ is

$$
u_{\gamma_{1}, \infty}(t)= \begin{cases}\gamma_{1} t, & \text { if } t \geq 0 \\ -\infty, & \text { if } t<0\end{cases}
$$

and $u_{1,1}(t)=t$

On the other hand, the OIC $\eta_{\gamma_{1}, \gamma_{2}}^{*}$ for any $\left(\gamma_{1}, \gamma_{2}\right) \in \Gamma$ is obtained in the next proposition, saying that $\eta_{\gamma_{1}, \gamma_{2}}^{*}$ is represented by the VaR.

Proposition 2.2 (Example 2.3 in Ben-Tal and Teboulle, 2007). For any $\left(\gamma_{1}, \gamma_{2}\right) \in \Gamma$, the OIC $\eta_{\gamma_{1}, \gamma_{2}}^{*}$ is given by

$$
\eta_{\gamma_{1}, \gamma_{2}}^{*}(X)=F_{X}^{-1}(\delta)=F_{X}^{-1}\left(\frac{1-\gamma_{1}}{\gamma_{2}-\gamma_{1}}\right),
$$

which coincides with the usual VaR multiplied by -1 .

It is clear that the OIC $\eta_{\gamma_{1}, \gamma_{2}}^{*}$ is positive homogeneous for any $\left(\gamma_{1}, \gamma_{2}\right) \in \Gamma$. However, it is not concave, implying that the OIC $\eta_{\gamma_{1}, \gamma_{2}}^{*}$ cannot be a monetary utility function. As we show in Sections 3 and 4, a sufficient condition for $\eta_{u}^{*}$ to be a law-invariant monetary utility function is the convexity of $u^{\prime}$. In the case of a piecewise linear utility, the first derivative of the utility function $u_{\gamma_{1}, \gamma_{2}}^{\prime}$ is not convex on $\mathbb{R} \backslash\{0\}$. Proposition 2.2 gives a counterexample for the OIC to be a monetary utility function.

The next proposition presents an important property of $\eta_{\gamma_{1}, \gamma_{2}}^{*}$. We derive a sufficient condition for the first-order stochastic dominance.

Proposition 2.3. If $\eta_{\gamma_{1}, \gamma_{2}}^{*}(X) \leq \eta_{\gamma_{1}, \gamma_{2}}^{*}(Y)$ for any $\left(\gamma_{1}, \gamma_{2}\right) \in \Gamma$, then $F_{X}(t) \leq F_{Y}(t)$ for any $t \in \mathbb{R}$.

Proof. For any $\delta \in(0,1)$, we can find a pair $\left(\gamma_{1}, \gamma_{2}\right) \in \Gamma$ such that $\delta=\frac{1-\gamma_{1}}{\gamma_{2}-\gamma_{1}}$. Thus, Proposition 2.3 immediately follows from Proposition 2.2.

Remark 2.4. For any $\gamma_{1} \in[0,1)$, the OIC $\eta_{\gamma_{1}, \infty}^{*}$ corresponding to $S_{\gamma_{1}, \infty}$ is essinf $X$. In this case, $\eta_{\gamma_{1}, \infty}^{*}$ is apparently a law-invariant coherent monetary utility function.

Remark 2.5. We exclude the case $\left(\gamma_{1}, \gamma_{2}\right)=(1,1)$ in our analysis because $\eta_{1,1}^{*}(X)=\infty$ for any $X \in L^{\infty}$ since $S_{1,1}(X)=t+\mathbb{E}\left[u_{1,1}(X-t)\right]$ for any $t \in \mathbb{R}$.

\section{OIC for Utility Functions whose Effective Domain is $\mathbb{R}$}

In this section, we investigate the properties of the OIC $\eta_{u}^{*}$, especially in relation to the functional form of the utility function $u$. First, we show that if $u^{\prime}$ is convex, the OIC $\eta_{u}^{*}$ is a law-invariant monetary utility function. Second, we prove that the OIC $\eta_{u}^{*}$ coincides with the OCE $S_{u}$ if and only if the Arrow-Pratt measure of absolute risk aversion is constant. 
Finally, we find that if $u^{\prime}$ is convex, the following conditions are equivalent: (1) the OCE $S_{u}$ is coherent, (2) the OIC $\eta_{u}^{*}$ is coherent, and (3) $u(t)=t$ for any $t>0$.

Throughout the section, we consider the case where the effective domain of the utility functions is the whole real line $\mathbb{R}$. In addition, we impose the following assumption.

Assumption 3.1. Any utility function $u$ appearing in this section is an $\mathbb{R}$-valued $C^{1}$-class function such that $u(t)<t$ for any $t<0$.

First, we provide the following proposition.

Proposition 3.2. For any $X \in L^{\infty}$, the OIC $\eta_{u}^{*}(X)$ satisfies the following properties:

(i) $S_{u}(X)=\eta_{u}^{*}(X)+\mathbb{E}\left[u\left(X-\eta_{u}^{*}(X)\right)\right]$.

(ii) $\eta_{u}^{*}(X) \in[\operatorname{essinf} X, \operatorname{esssup} X]$.

(iii) $\mathbb{E}\left[u^{\prime}\left(X-\eta_{u}^{*}(X)\right)\right]=1$.

Proof. For any $X \in L^{\infty}$, we define a function $g_{X}(t)$ by

$$
g_{X}(t)=t+\mathbb{E}[u(X-t)]
$$

Then, since $u$ is $C^{1}$-class and $X \in L^{\infty}, g_{X}$ is continuous, and

$$
g_{X}^{\prime}(t)=1-\mathbb{E}\left[u^{\prime}(X-t)\right]
$$

Note that $g_{X}^{\prime}$ is a non-increasing continuous function due to the continuity of $u^{\prime}$. Since $u^{\prime}(t) \leq 1$ for any $t \geq 0$, it holds that $g_{X}^{\prime}(\operatorname{essinf} X) \geq 0$. On the other hand, since $u^{\prime}(t)>1$ for any $t<0$ by Assumption 3.1, it holds that $g_{X}^{\prime}(\operatorname{esssup} X) \leq 0$. Therefore, because of the continuity of $g_{X}^{\prime}$, there exists a $t \in[\operatorname{essinf} X$, esssup $X]$ such that $g_{X}^{\prime}(t)=0$ from the Intermediate Value Theorem. Furthermore, there exists a maximum value $t$ such that $g_{X}^{\prime}(t)=0$, which gives $\eta_{u}^{*}(X)$. Thus, all of the claims are proven.

Remark 3.3. A similar result to Proposition 3.2 is obtained by Ben-Tal and Teboulle (1986) in Section 6 of their paper. However, we impose slightly different conditions on utility functions $u$. For example, Ben-Tal and Teboulle (1986) assume that $u$ is strictly concave, which is also mentioned in Remark 2.1 of Ben-Tal and Teboulle (2007). Proposition 2.1 of Ben-Tal and Teboulle (2007) should also be referred.

We present the following corollary, which is needed to prove that the OIC $\eta_{u}^{*}$ is a law-invariant monetary utility function.

Corollary 3.4. The OIC $\eta_{u}^{*}$ has the following representation:

$$
\eta_{u}^{*}(X)=\sup \left\{t \in \mathbb{R} \mid \mathbb{E}\left[u^{\prime}(X-t)\right] \leq 1\right\}
$$


Proof. This claim follows from Proposition 3.2 and the fact that $u^{\prime}$ is non-increasing.

Remark 3.5. (3.2) indicates that the OIC coincides with the u-mean certainty equivalent defined in (5.5) of Ben-Tal and Teboulle (2007) for the case where the corresponding utility function is given by $1-u^{\prime}(x)$.

As we explained in the introductory section, the OIC is especially important in the insurance industry. Let $X$ be a random variable representing how much money the insurance company pays to the purchaser, and let $f: \mathbb{R} \rightarrow \mathbb{R}$ be the function defined by $f(t)=1-u^{\prime}(-t)$, which is a non-increasing concave function with $f(0)=0$. Then, we have the representation

$$
-\eta_{u}^{*}(-X)=\inf \{t \in \mathbb{R} \mid \mathbb{E}[f(X-t)] \geq 0\},
$$

which indicates that $-\eta_{u}^{*}(-X)$ gives the least acceptable insurance premium for the insurance company. Siminarly, we can interpret that $\eta_{u}^{*}(X)$ is the greatest acceptable insurance premium for the insurance policy purchaser. Noting that $\eta_{u}^{*}(X)+\eta_{u}^{*}(-X) \leq 2 \eta_{u}^{*}(0)=0$ by the concavity of $\eta_{u}^{*}$, we can say that the interval $\left[\eta_{u}^{*}(X),-\eta_{u}^{*}(-X)\right]$ gives a pricing bound of $X{ }^{6}$

Furthermore, (3.2) is a similar form to the utility-based shortfall risk discussed in Section 4.9 of Föllmer and Schied (2016). Therefore, if $u^{\prime}$ is convex, $\eta_{u}^{*}$ is concave. Keeping this in mind, we obtain the following theorem.

Theorem 3.6. If $u^{\prime}$ is convex, then the OIC $\eta_{u}^{*}$ is a law-invariant monetary utility function.

Proof. It is apparent that $\eta_{u}^{*}$ is law-invariant by definition. The monotonicity and translation-invariance of $\eta_{u}^{*}$ easily follow from (3.2). Therefore it suffices to show that $\eta_{u}^{*}$ is concave to prove the theorem. Let $X$ and $Y$ be bounded random variables, and two real numbers $t_{X}$ and $t_{Y}$ be arbitrarily fixed such that $t_{X}<\eta_{u}^{*}(X)$ and $t_{Y}<\eta_{u}^{*}(Y)$, respectively. Since $u^{\prime}$ is non-increasing, it holds that

$$
\mathbb{E}\left[u^{\prime}\left(X-t_{X}\right)\right] \leq 1, \text { and } \mathbb{E}\left[u^{\prime}\left(Y-t_{Y}\right)\right] \leq 1 .
$$

Since $u^{\prime}$ is convex, it follows that for any $\lambda \in[0,1]$,

$$
\mathbb{E}\left[u^{\prime}\left(\lambda X+(1-\lambda) Y-\lambda t_{X}-(1-\lambda) t_{Y}\right)\right] \leq 1 .
$$

Therefore, we have

$$
\lambda t_{X}+(1-\lambda) t_{Y} \leq \eta_{u}^{*}(\lambda X+(1-\lambda) Y)
$$

showing that $\eta_{u}^{*}$ is concave.

\footnotetext{
${ }^{6}$ For more details on this matter, see Arai and Fukasawa (2014).
} 
Remark 3.7. The OIC $\eta_{u}^{*}$ is essentially the same as the notion of the modified prudence premium defined in Section 3 of Eeckhoudt et al. (2016). Let u be a $C^{3}$-class function. Then, the first-order condition implies

$$
\mathbb{E}\left[u^{\prime}\left(X-\eta_{u}^{*}(X)\right)\right]=1 .
$$

On the other hand, the equivalent precautionary premium associated with the random variable X, originally proposed by Kimball (1990) and here denoted by $\psi_{u}(X)$, is defined by

$$
\mathbb{E}\left[u^{\prime}(X)\right]=u^{\prime}\left(\mathbb{E}[X]-\psi_{u}(X)\right)
$$

Combining these two equations, we obtain

$$
\mathbb{E}\left[u^{\prime}\left(X-\eta_{u}^{*}(X)\right)\right]=u^{\prime}\left(\mathbb{E}[X]-\eta_{u}^{*}(X)-\psi_{u}\left(X-\eta_{u}^{*}(X)\right)\right)=1 .
$$

Roughly speaking, it follows from $\partial u(0)=1$ that

$$
\eta_{u}^{*}(X)=\mathbb{E}[X]-\psi_{u}\left(X-\eta_{u}^{*}(X)\right)
$$

The above equation implies that the decision maker evaluates the payoff $X$ by subtracting the precautionary premium from its expectation. The first-order approximation of $\psi_{u}$ by Kimball (1990) provides that $\psi_{u}\left(X-\eta_{u}^{*}(X)\right)$ is approximately equal to

$$
\frac{\operatorname{Var}(X)}{2} \lambda\left(\mathbb{E}\left[X-\eta_{u}^{*}(X)\right]\right)
$$

where $\lambda$ is the absolute prudence defined by

$$
\lambda(t)=-\frac{u^{\prime \prime \prime}(t)}{u^{\prime \prime}(t)} .
$$

This observation indicates that the OIC is the monetary utility function that reflects the precautionary demand for savings. Note that the precautionary premium increases if $u^{\prime}$ is more convex. Thus the third derivative of $u$ is of great significance in determining the OIC.

The next proposition shows that the OIC actually accounts for the risk of a random payoff by evaluating the payoff as lower than its expectation.

Proposition 3.8. If $u^{\prime}$ is convex, then $\eta_{u}^{*}(X) \leq \mathbb{E}[X]$ for any $X \in L^{\infty}$.

Proof. Since $u^{\prime}(t)>1$ for any $t<0$ by Assumption 3.1, it follows that for any $t>$ $\mathbb{E}[X], g_{X}^{\prime}(t)=1-\mathbb{E}\left[u^{\prime}(X-t)\right] \leq 1-u^{\prime}(\mathbb{E}[X]-t)<0$. Therefore, it holds that $\eta_{u}^{*}(X) \leq$ $\mathbb{E}[X]$.

Here, as an example of the utility functions used in economics and finance, we consider exponential utility functions. 
Example 3.9 (Exponential utility). For $c>0$, consider the following utility function:

$$
u(t)=\frac{1}{c}\left(1-e^{-c t}\right) .
$$

We call (3.3) the exponential utility functions with the Arrow-Pratt measure of absolute risk aversion c. We can easily check that exponential utility function satisfies Assumptions 2.1 and 3.1. Moreover, it is clear that $u^{\prime}$ is convex. As in Example 2.1 of Ben-Tal and Teboulle (2007), the OCE $S_{u}$ corresponding to the exponential utility functions is

$$
S_{u}(X)=-\frac{1}{c} \log \mathbb{E}\left[e^{-c X}\right],
$$

which is equal to the entropic risk measure multiplied by -1 as in Equation (4.12) of Föllmer and Schied (2016).

For exponential utility functions, the OIC $\eta_{u}^{*}$ coincides with $S_{u}$. Indeed, as Proposition 3.10 shows, the exponential utility is the only case where $S_{u}=\eta_{u}^{*}$ holds.

Proposition 3.10. If $u^{\prime}$ is convex and $S_{u}=\eta_{u}^{*}$, then $u$ is an exponential utility function defined by (3.3).

Proof. Assume that $S_{u}=\eta_{u}^{*}$. It follows from Proposition 3.2 that

$$
\left\{\begin{array}{l}
\mathbb{E}\left[u\left(X-\eta_{u}^{*}(X)\right)\right]=0, \\
\mathbb{E}\left[u^{\prime}\left(X-\eta_{u}^{*}(X)\right)\right]=1 .
\end{array}\right.
$$

As we show in Lemma 3.11 following this proposition, for any positive numbers $a$ and $b$, we can find a random variable $X$ that takes only two values $-a$ and $b$, and satisfies $S_{u}(X)=\eta_{u}^{*}(X)=0$. Let $p:=\mathbb{P}(X=b) \in(0,1)$. Then, from (3.4), we have

$$
\left\{\begin{array}{l}
(1-p) u(-a)+p u(b)=0 \\
(1-p) u^{\prime}(-a)+p u^{\prime}(b)=1
\end{array}\right.
$$

for the random variable $X$. Since $u(-a)<0, u(b)>0, u^{\prime}(-a)>1$, and $0 \leq u^{\prime}(b) \leq 1$ by the conditions imposed on $u$, it holds that

$$
\frac{1-u^{\prime}(-a)}{u(-a)}=\frac{1-u^{\prime}(b)}{u(b)} .
$$

Hence, since $a$ and $b$ are taken arbitrarily, there exists a positive real number $c>0$ such that

$$
c=\frac{1-u^{\prime}(t)}{u(t)}
$$

for any $t \in \mathbb{R} \backslash\{0\}$. Under the initial condition that $u(0)=0$, the differential equation (3.5) has a unique solution, which is given by the exponential utility function defined by (3.3).

The proof of Proposition 3.10 is completed if we show the following lemma. 
Lemma 3.11. Let $u$ be a utility function satisfying that $u^{\prime}$ is convex and $S_{u}=\eta_{u}^{*}$. For any $a>0$ and $b>0$, there exists a random variable $X$ that takes only two values $-a$ and $b$, and satisfies $\eta_{u}^{*}(X)=0$.

Proof. There are three steps for the proof.

Step 1. We see that $\operatorname{essinf} X<\eta_{u}^{*}(X)<\operatorname{esssup} X$ for any non-constant random variable $X \in L^{\infty}$. Assuming $\eta_{u}^{*}(X)=\operatorname{esssup} X$, we have $X-\eta_{u}^{*}(X) \leq 0$, which is equivalent to $u\left(X-\eta_{u}^{*}(X)\right) \leq 0$. Noting that $\mathbb{E}\left[u\left(X-\eta_{u}^{*}(X)\right)\right]=0$ by (3.4), and $u(t)<0$ for any $t<0$, we have $X-\eta_{u}^{*}(X)=0$, that is, $X-\operatorname{esssup} X=0$. This is a contradiction. Next, suppose that $\eta_{u}^{*}(X)=\operatorname{essinf} X$. Since $u^{\prime}(0)=1$ and $u(0)=0$, we have $u(t)>0$ for any $t>0$. Thus, a similar argument to the above leads to the fact that $X=\operatorname{essinf} X$, which is also a contradiction. As a result, we conclude that $\operatorname{essinf} X<\eta_{u}^{*}(X)<\operatorname{esssup} X$.

Step 2. We prove that $u^{\prime}(t)<1$ for any $t>0$. To this end, we assume $u^{\prime}(t)=1$ for some $t>0$. It holds then that $u^{\prime}(t)=1$ for any $t \geq 0$ by the convexity of $u^{\prime}$. Thus, together with (3.4), we have

$$
\begin{aligned}
1 & =\mathbb{E}\left[u^{\prime}\left(X-\eta_{u}^{*}(X)\right)\right] \\
& =\mathbb{P}\left(X-\eta_{u}^{*}(X) \geq 0\right)+\mathbb{E}\left[u^{\prime}\left(X-\eta_{u}^{*}(X)\right) \mathbf{1}_{\left\{X-\eta_{u}^{*}(X)<0\right\}}\right] \\
& >\mathbb{P}\left(X-\eta_{u}^{*}(X) \geq 0\right)+\mathbb{P}\left(X-\eta_{u}^{*}(X)<0\right)=1
\end{aligned}
$$

for any non-constant random variable $X \in L^{\infty}$, which is a contradiction. The inequality above is given from Step 1 and the fact that $u^{\prime}(t)>1$ for any $t<0$.

Step 3. For any $a>0$ and $b>0$, we take a random variable $X$ distributed as

$$
\mathbb{P}(X=b)=\frac{u^{\prime}(-a)-1}{u^{\prime}(-a)-u^{\prime}(b)}, \quad \mathbb{P}(X=-a)=1-\mathbb{P}(X=b) .
$$

We can immediately see that $\mathbb{P}(X=b) \in(0,1)$ by Step 2 , and $\mathbb{E}\left[u^{\prime}(X)\right]=1$, implying that $\eta_{u}^{*}(X)=0$.

Remark 3.12. Proposition 3.10 says that the OIC $\eta_{u}^{*}$ coincides with the OCE $S_{u}$ if and only if $u$ is a solution to the differential equation (3.5). Roughly speaking, this result is equivalent to the statement that the Arrow-Pratt measure of absolute risk aversion is constant. This is because $-u^{\prime \prime} / u^{\prime}=c$ follows from rewriting (3.5) as $c u=1-u^{\prime}$ and differentiating both sides of the equation.

Now we discuss the OIC in relation to certainty equivalent. Proposition 1 of Ben-Tal and Teboulle (1986) enumerates basic properties of the certainty equivalent $C_{u}$ as follows:

(a) $C_{u}$ is consistent, that is, $C_{u}(t)=t$ for any $t \in \mathbb{R}$.

(b) $C_{u}$ is invariant to any positive affine transformation in $u$, that is, $C_{a u+b}=C_{u}$ for any $a>0$ and $b \in \mathbb{R}$.

(c) $C_{u}(X) \leq \mathbb{E}[X]$ with equality for any $X \in L^{\infty}$ if and only if $u$ is linear. 
Condition (a) is equivalent to the condition given in Assumption 3.1 of the current study. That is, $u(t)<t$ for any $t<0$. Moreover, Proposition 3.2 (iii), which holds under Assumption 3.1, is roughly corresponding to condition (b), since Proposition 3.2 (iii) can be rewritten as $\mathbb{E}\left[u^{\prime}\left(X-\eta_{u}^{*}(X)\right)\right]=u^{\prime}(0){ }^{7}$ The first part of condition (c) is given in Proposition 3.8 of the current study. For the second part, our setting on utility functions excludes any linear function. However, in the limiting case where $c$ in (3.3) converges to 0 , the OIC for exponential utility converges to $\mathbb{E}[X]$. Note that $\eta_{u}^{*}(X)=\mathbb{E}[X]$ if and only if the precautionary premium defined in Remark 3.7 is equal to zero, that is, $\psi_{u}\left(X-\eta_{u}^{*}(X)\right)=0$. As the curvature of $u$ is becoming linear, $\psi_{u}$ tends to zero. This implies that the convexity of $u^{\prime}$ is becoming weak, which coincides with comments in Remark 3.7.

Next, we consider two types of quadratic utility functions, and discuss the OIC's coherency under our settings.

Example 3.13 (Quadratic utility 1). Suppose that the utility function is of the quadratic form presented in Example 2.2 of Ben-Tal and Teboulle (1986):

$$
u(t)= \begin{cases}t-\frac{1}{2} t^{2}, & \text { if } t<1, \\ \frac{1}{2}, & \text { otherwise. }\end{cases}
$$

As Ben-Tal and Teboulle (1986) show, $S_{u}(X)=\mathbb{E}[X]-\operatorname{Var}(X) / 2$ and $\eta_{u}^{*}(X)=\mathbb{E}[X]$ if $\operatorname{esssup} X \leq \mathbb{E}[X]+1$

Example 3.14 (Quadratic utility 2). Suppose that the utility function is of the quadratic form

$$
u(t)= \begin{cases}t-c t^{2}, & \text { if } t<0 \\ t, & \text { otherwise }\end{cases}
$$

where $c>0$. Then we have

$$
u^{\prime}(t)= \begin{cases}1-2 c t, & \text { if } t<0, \\ 1, & \text { otherwise, }\end{cases}
$$

and that $u^{\prime}$ is convex. Here, we obtain $S_{u}$ as

$$
\begin{aligned}
S_{u}(X) & =\sup \{t \in \mathbb{R} \mid t+\mathbb{E}[u(X-t)]\} \\
& =\sup \left\{t \in \mathbb{R} \mid t+\mathbb{E}\left[X-t-c(X-t)^{2} \mathbf{1}_{\{X \leq t\}}\right]\right\} \\
& =\mathbb{E}[X]-c \inf \left\{t \in \mathbb{R} \mid \mathbb{E}\left[(X-t)^{2} \mathbf{1}_{\{X \leq t\}}\right]\right\} .
\end{aligned}
$$

The infimum in the last line of the equation above should be equal to zero since $\mathbb{E}[(X-$ $\left.t)^{2} \mathbf{1}_{\{X \leq t\}}\right]$ is positive for any $t>\operatorname{essinf} X$ and zero for any $t \leq \operatorname{essinf} X$. Thus, $S_{u}(X)=$ $\mathbb{E}[X]$. Substituting this into the equation given in Proposition 3.2 (i) implies that

$$
\eta_{u}^{*}(X)=\operatorname{essinf} X
$$

We thus easily verify that $\eta_{u}^{*}$ is coherent.

\footnotetext{
${ }^{7}$ Strictly speaking, this argument is not necessarily exact because any positive affine transformation of $u$ is no longer a utility function in our setting. Note that $u$ and $a u+b$ describe the same preference in the expected utility framework.
} 
Remark 3.15. The OCE $S_{u}$ corresponding to the utility function u defined by (3.6) is also coherent. Note that $S_{u}$ and $\eta_{u}^{*}$ do not depend on the value of $c$.

We can generalize the result in Remark 3.15 by a similar argument. That is, the OCE $S_{u}$ and the OIC $\eta_{u}^{*}$ are coherent under some conditions for utility functions $u$. We omit the proof since it is an easy exercise.

Proposition 3.16. For any utility function $u$ such that $u^{\prime}$ is convex and $u(t)=t$ for any $t>0$, we have that $S_{u}(X)=\mathbb{E}[X]$ and $\eta_{u}^{*}(X)=\operatorname{essinf} X$. In particular, $S_{u}$ and $\eta_{u}^{*}$ are coherent.

In addition, we obtain the converse of Proposition 3.16.

Proposition 3.17. Suppose that $u^{\prime}$ is convex. If $\eta_{u}^{*}$ is coherent, then $\eta_{u}^{*}(X)=\operatorname{essinf} X$, $S_{u}(X)=\mathbb{E}[X]$ and $u(t)=t$ for any $t>0$.

Proof. Denoting $h(t):=u^{\prime}(t)-1$, we can rewrite (3.2) as

$$
\eta_{u}^{*}(X)=\sup \{t \in \mathbb{R} \mid \mathbb{E}[h(X-t)] \leq 0\} .
$$

It suffices to show that $h(t)=0$ for any $t>0$. By the conditions on $u, h$ is a nonincreasing continuous convex function satisfying $h(0)=0, h(t) \geq-1$ for any $t \in \mathbb{R}$, and $\lim _{t \rightarrow-\infty} h(t)=\infty$. Moreover, if there is a $t>0$ such that $h(t)<0$, then $h(t)<0$ for any $t>0$.

Now, suppose that $h(t)<0$ for any $t>0$. Taking $a>0$ and $b>0$ arbitrarily, we define a random variable $X$ with the following distribution

$$
\mathbb{P}(X=b)=\frac{h(-a)}{h(-a)-h(b)}(=: p), \text { and } \mathbb{P}(X=-a)=\frac{-h(b)}{h(-a)-h(b)} .
$$

Note that $h(-a)>0$ and $p \in(0,1)$. We have then $\mathbb{E}[h(X)]=0$, from which $\eta_{u}^{*}(X)=0$ follows. On the other hand, the coherency of $\eta_{u}^{*}$ implies that $p h(\lambda b)+(1-p) h(-\lambda a)=0$ for any $\lambda>0$. However, this is a contradiction, since $h(\lambda b)>-1$ for any $\lambda>0$ and $\lim _{\lambda \rightarrow \infty} h(-\lambda a)=\infty$. Consequently, $h(t)=0$ for any $t>0$.

From the above two propositions, supposing that $u^{\prime}$ is convex, we have the equivalence between the coherency of $\eta_{u}^{*}$ and the condition that $u(t)=t$ for any $t>0$. In particular, Proposition 3.17 ensures that $S_{u}$ is coherent whenever $\eta_{u}^{*}$ is coherent. Now, we see the converse of this inclusion relation as follows.

Proposition 3.18. Suppose that $u^{\prime}$ is convex. If $S_{u}$ is coherent, then $\eta_{u}^{*}(X)=\operatorname{essinf} X$, $S_{u}(X)=\mathbb{E}[X]$ and $u(t)=t$ for any $t>0$.

Proof. $\quad$ From the view of Proposition 3.16, it suffices to show that $u(t)=t$ for any $t>0$ if $S_{u}$ is coherent. Assume that there is a $t>0$ such that $u(t)<t$. Then, the convexity of $u^{\prime}$ implies that $u(t)<t$ for any $t>0$, that is, $u(t)<t$ for any $t \in \mathbb{R} \backslash\{0\}$. By Theorem 
3.1 of Ben-Tal and Teboulle (2007), $S_{u}$ is not coherent, since $u^{\prime}$ is never convex when $u$ is a piecewise linear function. This completes the proof of the proposition.

In summary, when $u^{\prime}$ is convex, the following conditions are equivalent: (1) $S_{u}$ is coherent, (2) $\eta_{u}^{*}$ is coherent, and (3) $u(t)=t$ for any $t>0$. In particular, when one of the above three conditions holds, $S_{u}(X)$ and $\eta_{u}^{*}(X)$ are given by $\mathbb{E}[X]$ and essinf $X$, respectively. This observation implies that the decision maker optimally reserves the initial capital to cover the highest potential losses. In other words, the decision maker is significantly risk-averse and takes the worst case scenario into account to set the initial capital.

\section{OIC for Logarithmic Utility and Power Utility Func- tions}

In the previous section, we analyze the case in which the effective domain is $\mathbb{R}$. In this section, as examples where the effective domain is a half line, we consider logarithmic utility and power utility functions, and show that the OIC for such utility functions is also a law-invariant monetary utility function. This result corresponds to Theorem 3.6 in the previous section.

For $\gamma>0$, define the function $u_{\gamma}: \mathbb{R} \rightarrow[-\infty,+\infty)$ by

$$
u_{\gamma}(t)= \begin{cases}\frac{(1+t)^{1-\gamma}-1}{1-\gamma}, & \text { if } \gamma \neq 1 \text { and } t>-1 \\ \log (1+t), & \text { if } \gamma=1 \text { and } t>-1 \\ \frac{-1}{1-\gamma}, & \text { if } 0<\gamma<1 \text { and } t=-1 \\ -\infty, & \text { otherwise. }\end{cases}
$$

The utility function $u_{\gamma}$ is called power utility for $\gamma \neq 1$, and logarithmic utility for $\gamma=1$. Note that for each $t>-1$, it holds that

$$
u_{1}(t)=\lim _{\gamma \rightarrow 1} u_{\gamma}(t)
$$

Moreover, the effective domain for $u_{\gamma}$ is $(-1, \infty)$ for $\gamma \geq 1$, and is $[-1, \infty)$ for $0<\gamma<1$, respectively. The utility function $u_{\gamma}$ is a $C^{1}$-class function on $(-1, \infty)$, and $u_{\gamma}^{\prime}$ is provided by

$$
u_{\gamma}^{\prime}(t)=(1+t)^{-\gamma}
$$

for $t \in(-1, \infty)$, which is a convex function. In addition, we define $u_{\gamma}^{\prime}(-1):=+\infty$ for convenience. 
Next, for the function $u_{\gamma}$, we present results similar to Proposition 3.2 and Theorem 3.6 in the former section. Here, for each $\gamma>0$, we denote the corresponding OCE and OIC by $S_{\gamma}$ and $\eta_{\gamma}^{*}$, respectively. First, similar to (3.1), we define the function $g_{X}: \mathbb{R} \rightarrow$ $[-\infty,+\infty)$ by

$$
g_{X}(t)=t+\mathbb{E}\left[u_{\gamma}(X-t)\right]
$$

Then, $g_{X}$ is a $C^{1}$-class function on $(-\infty, \operatorname{essinf} X+1)$, and satisfies

$$
g_{X}^{\prime}(t)=1-\mathbb{E}\left[u_{\gamma}^{\prime}(X-t)\right]
$$

for $t \in(-\infty, \operatorname{essinf} X+1)$. Note that $g_{X}^{\prime}$ is non-increasing, and for $t \leq \operatorname{essinf} X$, it holds that

$$
g_{X}^{\prime}(t) \geq 1-u_{\gamma}^{\prime}(0)=0 .
$$

For the analysis below, we decompose $L^{\infty}$ into the following two classes:

$$
\begin{aligned}
& A_{1}:=\left\{X \in L^{\infty} \mid g_{X}^{\prime}(t)<0 \text { for some } t \in(-\infty, \operatorname{essinf} X+1)\right\}, \\
& A_{2}:=\left\{X \in L^{\infty} \mid g_{X}^{\prime}(t) \geq 0 \text { for any } t \in(-\infty, \operatorname{essinf} X+1)\right\} .
\end{aligned}
$$

Before presenting the main theorem, we show the following results.

Lemma 4.1. For any $X \in A_{2}$, we have

$$
\lim _{t \uparrow e s s i n f X+1} g_{X}(t)=g_{X}(\operatorname{essinf} X+1) \in \mathbb{R} .
$$

Proof. This is obvious for the case of $0<\gamma<1$. Thus, we only consider the case $\gamma \geq 1$. For any $X \in A_{2}$, we have $\mathbb{E}\left[u_{\gamma}^{\prime}(X-t)\right] \in[0,1]$ for any $t \in(-\infty, \operatorname{essinf} X+1)$. Since $u_{\gamma}^{\prime}(X-t)$ is non-decreasing as a function of $t$, the monotone convergence theorem, together with the right-continuity of $u_{\gamma}^{\prime}$ on $[-1, \infty)$, provides that

$$
1 \geq \lim _{t \uparrow \operatorname{essinf} X+1} \mathbb{E}\left[u_{\gamma}^{\prime}(X-t)\right]=\mathbb{E}\left[u_{\gamma}^{\prime}(X-\operatorname{essinf} X-1)\right] .
$$

Consequently, we have $\mathbb{P}(X=\operatorname{essinf} X)=0$. Now, for any $t \in(-1,0)$, it holds that $(1+t)^{1-\gamma}<(1+t)^{-\gamma}$ for $\gamma>1$, and $-\log (1+t)<(1+t)^{-1}$, indicating that $-u_{\gamma}(X-$ essinf $X-1)$ is also integrable. Then, using the monotone convergence theorem again, we obtain

$$
\lim _{t \uparrow \operatorname{essinf} X+1} \mathbb{E}\left[-u_{\gamma}(X-t)\right]=\mathbb{E}\left[-u_{\gamma}(X-\operatorname{essinf} X-1)\right] \in \mathbb{R}
$$

Proposition 4.2. For any $\gamma>0$ in (4.1) and any $X \in L^{\infty}$, the OIC $\eta_{\gamma}^{*}(X)$ satisfies the following relations: 
1. $S_{\gamma}(X)=g_{X}\left(\eta_{\gamma}^{*}(X)\right)$,

2. for $X \in A_{1}, \eta_{\gamma}^{*}(X) \in[\operatorname{essinf} X, \operatorname{essinf} X+1)$, and for $X \in A_{2}, \eta_{\gamma}^{*}(X)=\operatorname{essinf} X+1$,

3. $\mathbb{E}\left[u_{\gamma}^{\prime}\left(X-\eta_{\gamma}^{*}(X)\right)\right] \leq 1$. If $X \in A_{1}, \mathbb{E}\left[u_{\gamma}^{\prime}\left(X-\eta_{\gamma}^{*}(X)\right)\right]=1$.

Proof. For $X \in A_{1}$, since $g_{X}^{\prime}$ is non-increasing, (4.2) implies that there exists a $t \in$ $[\operatorname{essinf} X, \operatorname{essinf} X+1)$ such that $g_{X}^{\prime}(t)=0$. Thus all of the claims in Proposition 4.2 follow for $X \in A_{1}$. For $X \in A_{2}$, since $g_{X}$ is non-decreasing on $(-\infty, \operatorname{essinf} X+1)$, Lemma 4.1 implies that all of the claims hold true, which completes the proof.

Corollary 4.3. If $\mathbb{E}[X]<\operatorname{essinf} X+1$, then $X \in A_{1}$, and $\eta_{\gamma}^{*}(X) \in[\operatorname{essinf} X, \mathbb{E}[X]]$.

Proof. Since $u_{\gamma}^{\prime}$ is convex, by taking a sufficiently small $\varepsilon>0$ such that $\mathbb{E}[X]+\varepsilon<$ essinf $X+1$, it holds that

$$
g_{X}^{\prime}(\mathbb{E}[X]+\varepsilon) \leq 1-u_{\gamma}^{\prime}(\mathbb{E}[X-\mathbb{E}[X]]-\varepsilon)=1-u_{\gamma}^{\prime}(-\varepsilon)<0 .
$$

Thus, $X \in A_{1}$. We can show the latter claim similarly.

Now we are ready to provide the main result of this section.

Theorem 4.4. The OIC $\eta_{\gamma}^{*}$ is a law-invariant monetary utility function.

Proof. By Proposition 4.2, it is clear that $\eta_{\gamma}^{*}$ is $\mathbb{R}$-valued. It is also clear that $\eta_{\gamma}^{*}$ is law-invariant. For any $c \in \mathbb{R}$ and $i=1,2$, if $X \in A_{i}$, then $X+c \in A_{i}$. Thus, $\eta_{\gamma}^{*}$ satisfies the translation-invariance.

Next, we show the monotonicity of $\eta_{\gamma}^{*}$. Take two random variables $X, Y \in L^{\infty}$ with $X \geq Y$. When $\eta_{\gamma}^{*}(X) \geq \operatorname{essinf} Y+1$, we have $\eta_{\gamma}^{*}(X) \geq \eta_{\gamma}^{*}(Y)$ since essinf $Y+1 \geq \eta_{\gamma}^{*}(Y)$. On the other hand, $g_{X}^{\prime}\left(\eta_{\gamma}^{*}(X)\right)=0$ holds when $\eta_{\gamma}^{*}(X)<\operatorname{essinf} Y+1$. Since $g_{X}^{\prime}(t) \geq g_{Y}^{\prime}(t)$ for any $t \in(-\infty$, essinf $Y+1)$, we have $g_{Y}^{\prime}\left(\eta_{\gamma}^{*}(X)\right) \leq 0$. Thus, we obtain $\eta_{\gamma}^{*}(X) \geq \eta_{\gamma}^{*}(Y)$, from which the monotonicity of $\eta_{\gamma}^{*}$ follows.

Finally, we show the concavity of $\eta_{\gamma}^{*}$. Let $X, Y \in L^{\infty}$, and let $\lambda \in[0,1]$. If $\lambda X+(1-$ $\lambda) Y \in A_{2}$, we have

$$
\begin{aligned}
\eta_{\gamma}^{*}(\lambda X+(1-\lambda) Y) & =\operatorname{essinf}(\lambda X+(1-\lambda) Y)+1 \\
& \geq \lambda(\operatorname{essinf} X+1)+(1-\lambda)(\operatorname{essinf} Y+1) \\
& \geq \lambda \eta_{\gamma}^{*}(X)+(1-\lambda) \eta_{\gamma}^{*}(Y)
\end{aligned}
$$

which shows the concavity. In the case where $\lambda X+(1-\lambda) Y \in A_{1}$, take $t_{X}<\eta_{\gamma}^{*}(X)$ and $t_{Y}<\eta_{\gamma}^{*}(Y)$, arbitrarily. Then, since $\mathbb{E}\left[u_{\gamma}^{\prime}\left(X-t_{X}\right)\right] \leq 1$, it follows that

$$
1 \geq \lambda \mathbb{E}\left[u_{\gamma}^{\prime}\left(X-t_{X}\right)\right]+(1-\lambda) \mathbb{E}\left[u_{\gamma}^{\prime}\left(Y-t_{Y}\right)\right] \geq \mathbb{E}\left[u_{\gamma}^{\prime}\left(\lambda X+(1-\lambda) Y-\lambda t_{X}-(1-\lambda) t_{Y}\right)\right]
$$

That is, $g_{\lambda X+(1-\lambda) Y}^{\prime}\left(\lambda t_{X}+(1-\lambda) t_{Y}\right) \geq 0$. On the other hand, noting that $\lambda X+(1-$ $\lambda) Y \in A_{1}$, we have $g_{\lambda X+(1-\lambda) Y}^{\prime}\left(\eta_{\gamma}^{*}(\lambda X+(1-\lambda) Y)\right)=0$. Thus, since $g_{\lambda X+(1-\lambda) Y}^{\prime}$ is 
non-increasing, it holds that $\eta_{\gamma}^{*}(\lambda X+(1-\lambda) Y) \geq \lambda t_{X}+(1-\lambda) t_{Y}$. By the arbitrariness of $t_{X}$ and $t_{Y}$, it follows that $\eta_{\gamma}^{*}(\lambda X+(1-\lambda) Y) \geq \lambda \eta_{\gamma}^{*}(X)+(1-\lambda) \eta_{\gamma}^{*}(Y)$.

Remark 4.5. The theorem above provides the same result as Theorem 3.6 for logarithmic and power utility functions, which are frequently used in economics and finance, but not analyzed in Ben-Tal and Teboulle (2007).

Example 4.6. Consider the logarithmic utility function $u(t)=\log (1+t)$. Let $X$ be $a$ random variable distributed uniformly on $[a, b]$, where $-1<a<b$. We have

$$
g^{\prime}(t)=1-\frac{1}{b-a} \log \left(1+\frac{b-a}{1+a-t}\right)
$$

for any $t<a+1$. For any small positive number $\varepsilon>0$ satisfying $1+\frac{b-a}{\varepsilon}>e^{b-a}$, we have

$$
g^{\prime}(a+1-\varepsilon)=1-\frac{1}{b-a} \log \left(1+\frac{b-a}{\varepsilon}\right)<0,
$$

meaning that $X \in A_{1}$. Moreover, we obtain

$$
\eta_{u}^{*}(X)=1+\frac{a e^{b}-b e^{a}}{e^{b}-e^{a}}
$$

\section{Conclusion}

In this paper, we propose the notion of OIC, induced by the OCE proposed by Ben-Tal and Teboulle (1986). By providing several properties of the OIC with different utility functions or other assumptions, we successfully present the OIC whose negative value is a good risk measure for the future risks with the decision-maker's concrete criteria in the background. One example is that the OIC becomes a law-invariant monetary utility function when the first derivative of the utility function is convex. This result implies that the monetary utility function is closely related with the prudence premium originally proposed by Kimball (1990).

There are several directions for future research. The first is an application to financial risks. For example, in terms of evaluating portfolio risk in the financial sector, the choice of function for the utility function $u$ is important. Second, future research should discuss the acceptance set of uncertain payoffs associated with utility $u$. Future research should aim to solve these practical problems, among others. 


\section{References}

Acerbi, C. and D. Tasche (2002), "On the Coherence of Expected Shortfall," Journal of Banking and Finance, 26(7), 1487-1503.

Arai, T. and M. Fukasawa (2014), "Convex Risk Measures for Good Deal Bounds," Mathematical Finance, 24, 464-484.

Artzner, P., F. Delbaen, J.-M. Eber, and D. Heath (1999), "Coherent Measures of Risk," Mathematical Finance, 9(3), 203-228.

Balbás, A., B. Balbás, and A. Heras (2009), "Optimal Reinsurance with General Risk Measures," Insurance: Mathematics and Economics, 44, 374-384.

Balbás, A., B. Balbás, R. Balbás, and A. Heras (2015), “Optimal Reinsurance under Risk and Uncertainty," Insurance: Mathematics and Economics, 60, 61-74.

Ben-Tal, A. and M. Teboulle (1986), "Expected Utility, Penalty Functions, and Duality in Stochastic Nonlinear Programming," Management Science, 32(11), 1445-1466.

Ben-Tal, A. and M. Teboulle (2007), "An Old-New Concept of Convex Risk Measures: The Optimized Certainty Equivalent," Mathematical Finance, 17(3), 449-476.

Boonen, T. J. (2015), “Competitive Equilibria with Distortion Risk Measures," Astin Bulletin, 45(3), 703-728.

Chen, W., M. Sim, J. Sun, and C.-P. Teo (2010), “CVaR to Uncertainty Set: Implications in Joint Chance-Constrained Optimization," Operations Research, 58(2), 470-485.

Cheridito, P. and T. Li (2009), "Risk Measures on Orlicz Hearts," Mathematical Finance, 19(2), 189-214.

Chi, Y. and K. S. Tan (2013), "Optimal Reinsurance with General Premium Principles," Insurance: Mathematics and Economics, 52, 180-189.

Drapeau, S. and M. Kupper (2013), "Risk Preferences and Their Robust Representation," Mathematics of Operations Research, 38(1), 28-62.

Eeckhoudt, L. R., E. Pagani, and E. R. Gianin (2016), "Prudence, Risk Measures and the Optimized Certainty Equivalent: A Note," preprint.

Föllmer, H. and A. Schied (2002), "Robust Preferences and Convex Measures of Risk," in Sandmann, K. and P. J. Schönbucher (eds.), Advances in Finance and Stochastics, Springer, 39-65.

Föllmer, H. and A. Schied (2016), Stochastic Finance: An Introduction in Discrete Time, 4 th edition, de Gruyter. 
Frittelli, M. and E. Rosazza Gianin (2002), "Putting Order in Risk Measures," Journal of Banking and Finance, 26(7), 1473-1486.

Goh, J. and M. Sim (2010), "Distributionally Robust Optimization and Its Tractable Approximations," Operations Research, 58(4-1), 902-917.

Kimball, M. S. (1990), "Precautionary Saving in the Small and in the Large," Econometrica, 58(1), 57-73.

Kong, D., L. Liu, and Y. Wu (2017), "Optimal Reinsurance under Risk and Uncertainty on Orlicz Hearts," forthcoming in Insurance: Mathematics and Economics.

Liebrich, F.-B. and G. Svindland (2017), "Model Spaces for Risk Measures," Insurance: Mathematics and Economics, 77, 150-165.

Pratt, J. W. (1964), "Risk Aversion in the Small and in the Large," Econometrica, 31(1-2), $122-136$.

Rieger, M. O. (2017), "Characterization of Acceptance Sets for Co-monotone Risk Measures," Insurance: Mathematics and Economics, 74, 147-152.

Schied, A., H. Föllmer, and S. Weber (2009), "Robust Preferences and Robust Portfolio Choice," in Mathematical Modelling and Numerical Methods in Finance Volume 15, 29-87.

Tan, K. S., C. Weng, and Y. Zhang (2011), "Optimality of General Reinsurance Contracts under CTE Risk Measure," Insurance: Mathematics and Economics, 49, 175-187. 